\title{
Time-dependent modelling of PKS 2155-304 in a low state: one- or two-zone emission modelling?
}

\author{
Maria Petropoulou1, a and Apostolos Mastichiadis ${ }^{1, b}$ \\ ${ }^{1}$ Department of Astrophysics, Astronomy and Mechanics \\ Faculty of Physics \\ University of Athens \\ Greece
}

\begin{abstract}
One-zone radiation models have been widely used in modelling the steady-state multiwavelength (MW) spectra of blazars, having as main goal the determination of the physical conditions in the emitting region, such as the magnetic field strength, the species of radiating particles etc. Then, the results from onezone stationary modelling are often used as a stepping stone for studying flaring events. Here we show that the application of steady-state one-zone models on intrinsic variable sources, even when these are in a low state, can be misleading. Although the one-zone SSC and proton synchrotron models succeed in fitting the time-averaged MW spectrum, they cannot easily (or at all) reproduce the small amplitude multifrequency variability. We show that a two-component leptonic model addresses both spectral and temporal observations more successfully, albeit at the expense of more free parameters.
\end{abstract}

\section{Introduction}

In the present work we attempt a time-dependent fit to the MW observations of blazar PKS 2155-304 at redshift $z=0.116$ [1]. This was observed, while being in a low state, in four energy bands (optical, $\mathrm{X}$-rays, $\mathrm{GeV}$ and $\mathrm{TeV}$ $\gamma$-rays). The observed variability was marginal implying that the physical conditions did not vary significantly. In order to fit the data we have applied three models of increasing complexity: (i) an one-component SSC model (1SSC), (ii) a leptohadronic proton synchrotron model (LHs) and (iii) a two-component SSC model (2-SSC). The details of the modelling and the subsequent results are presented in Sections 2 and 3, respectively. We conclude in Section 4 with a discussion of our results.

\section{Model description}

Although the method we follow is similar to that described in [2], it features time-dependent fitting using not only an SSC model but a leptohadronic one (LHs) as well; for an application of stationary solutions within leptohadronic models on MW spectra of PKS 2155-304, see [3]. Details about the numerical code we have used can be found in $[4,5]$. In all cases we have employed a two-step process:

1. We determine the parameter values that give an acceptable fit to the time-averaged SED of the source.

\footnotetext{
a. e-mail: maroulaaki@gmail.com
}

b. e-mail: amastich@phys.uoa.gr
2. We vary one or more parameters following the variability pattern observed in a specific energy band(s).

The amplitude of the parameter variations is determined by trial and error until an acceptable fit to one or more light curves is obtained. In all models the maximum Lorentz factor of electrons of the first component varied according to

$$
\gamma_{\max }^{e}=\left\langle\gamma_{\max }^{e}\right\rangle\left(\alpha_{1} \frac{F_{\mathrm{X}}(\tau)}{F_{\mathrm{X}}^{\max }}\right)^{\beta_{1}},
$$

where $F_{\mathrm{X}}^{\max }=9.8 \times 10^{-11} \mathrm{erg} \mathrm{cm}^{-2} \mathrm{~s}^{-1}$ is the maximum flux in the 2-10 keV energy band and $F_{X}(\tau)$ is the result of a cubic spline interpolation of the obsevred X-ray light curve expressed in terms of the dimensionless comoving time $\tau=t / t_{\mathrm{cr}}$, where $t_{\mathrm{cr}}=R / c$ with $R$ the size of the source. The transformation between the observed and comoving times has been made using the Doppler factor value determined by the time-averaged SED fitting. The first date of the observing campaign (MJD 54704) corresponds to $\tau=0$. Equation (1) assumes that the variations of $\gamma_{\max }^{e}$ are a scaled version of the observed X-ray variability. This scenario is adopted in all three models since it is physically motivated by the spectral hardening observed during episodes of increasing flux (see e.g. Fig. 1 in [1]). In the 2-SSC model the variations introduced for the second emitting component were modelled according to the optical flux variability, i.e.

$$
\gamma_{\max }^{e}=\left\langle\gamma_{e}^{\max }\right\rangle\left(\alpha_{2} \frac{F_{\mathrm{opt}}(\tau)}{F_{\mathrm{opt}}^{\max }}\right)^{\beta_{2}},
$$


Table 1. Parameter values for each of the three models discussed in text.

\begin{tabular}{ccccc}
\hline $\begin{array}{c}\text { Parameters for } \\
\text { variability }\end{array}$ & 1 -SSC & \multicolumn{2}{c}{2 -SSC } & LHs \\
\hline$\alpha_{1} / \beta_{1}$ & $1.5 / 0.7$ & $2.0,1.8$ & $2.0 / 1.8$ \\
$\alpha_{2} / \beta_{2}$ & - & $2.0,1.0$ & - \\
$f_{e} / f_{p}$ & - & \multicolumn{2}{c}{-} & $0.6 / 0.5$ \\
$g_{e} / g_{p}$ & - & & - & $0.0 / 0.5$ \\
\hline \hline Parameters for & & 1 st & 2 nd \\
average SED & & & & \\
\hline \hline$B(\mathrm{G})$ & 0.5 & 20 & 0.3 & 40 \\
$R(\mathrm{~cm})$ & $10^{16}$ & $3 \times 10^{15}$ & $1.5 \times 10^{16}$ & $10^{16}$ \\
$\delta$ & 34 & 18 & 34 & 28 \\
\hline$\gamma_{\min }^{e}$ & $10^{3.6}$ & $10^{3.8}$ & $10^{3.6}$ & $10^{3.0}$ \\
$\gamma_{\max }^{e}$ & $10^{5.3}$ & $10^{4.8}$ & $10^{4.3}$ & $10^{4.7}$ \\
$\ell_{e}$ & $10^{-4.3}$ & $10^{-3.5}$ & $10^{-4.2}$ & $10^{-4.35}$ \\
$p_{e}$ & 2.4 & 3.0 & 1.8 & 2.6 \\
\hline$\gamma_{\min }^{p}$ & - & - & - & $10^{7}$ \\
$\gamma_{\max }^{p}$ & - & - & - & $10^{9.9}$ \\
$\ell_{p}$ & - & - & - & $10^{-6.5}$ \\
$p_{p}$ & - & - & - & 2.4 \\
\hline
\end{tabular}

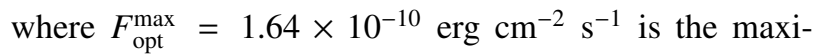
mum flux measured in the BV filters. We note also that the subscripts 1,2 of the constants $a, b$ appearing in above equations refer to the first and second components, respectively. The other parameters that we allowed to vary are the injection compactnesses of primary particles, i.e. electrons $\left(\ell_{e}\right)$ in the 1-SSC and 2-SSC models and both protons and electrons $\left(\ell_{e, p}\right)$ in the LHs model; here, the compactness is defined as

$$
\ell_{e, p}=\frac{L_{e, p}^{\mathrm{inj}} \sigma_{\mathrm{T}}}{4 \pi R m_{e, p} c^{3}},
$$

where $L_{e, p}^{\mathrm{inj}}$ is the total injected luminosity in electrons/protons and $\sigma_{\mathrm{T}}$ is the Thomson cross section. Moreover, the variations of the injection compactnesses follow the variability pattern observed in the optical bands:

$$
\ell_{e, p}=\left\langle\ell_{e, p}\right\rangle \frac{1}{f_{e, p}}\left(\frac{F_{\mathrm{opt}}}{F_{\mathrm{opt}}^{\max }}\right)+g_{e, p} .
$$

This particular choice is justified by the tight correlation between the optical and $\mathrm{TeV}$ fluxes, which in the LHs model are attributed to the synchrotron radiation of electrons and protons, respectively. The parameters $\alpha_{1,2}, \beta_{1,2}$, $f_{e, p}$ and $g_{e, p}$ are free and they are determined by finding the best fit to the light curves. The values used in our modelling are summarized in Table 1.

\section{Results}

In this section we present our main results, i.e. MW time-dependent spectra and light curves, for each of the models discussed in the previous section. We comment also on the advantages and disadvantages of each model.

\subsection{One-component SSC model}

The 1-SSC model describes fairly well the X-ray variability (see left bottom panel of Fig. 1) under the assumption of small amplitude variations of $\gamma_{\max }^{e}$, i.e.

$$
\frac{\delta \log \left(\gamma_{\max }^{e}\right)}{\left\langle\log \gamma_{\max }^{e}\right\rangle}=\frac{\max \left(\log \gamma_{\max }^{e}\right)}{\left\langle\log \gamma_{\max }^{e}\right\rangle}-1 \simeq 0.02 .
$$

However, the model predicts a tight correlation between the $\mathrm{X}$-ray and $\mathrm{TeV} \gamma$-ray fluxes which is not detected (see right bottom panel in Fig. 1). Moreover, the variation of $\gamma_{\max }^{e}$ alone cannot account for the observed optical variability (see top panel in Fig. 1). The combination of the steep electron spectrum at injection with the low value of the magnetic field result in an almost constant optical flux. If we had allowed $\ell_{e}$ to vary according to eq. (4), then larger variations of $\gamma_{\max }^{e}$ would be required, e.g. $\frac{\delta \log \left(\gamma_{\max }^{e}\right)}{\left\langle\log \gamma_{\max }^{e}\right\rangle}>0.1$, since the optical and X-ray fluxes are not correlated ${ }^{1}$. We note also that if the changes of $\gamma_{\max }^{e}$ become large enough one cannot avoid spectral variability in the $\mathrm{TeV}$ energy band even when Klein-Nishina effects are taken into account.

\subsection{Leptohadronic synchrotron (LHs) model}

In this model the low-energy bump (optical up to Xrays) is attributed to the synchrotron radiation of primary electrons whereas the high-energy bump is considered to be synchrotron emission from a relativistic proton distribution present in the same region. Thus, the number of free parameters required to describe the time-averaged SED increases significantly from seven, in the 1-SSC model, to eleven. Similar to the 1-SSC model, we varied $\gamma_{\max }^{e}$ (see eq. (1)) in order to account for the X-ray flux and spectral variability. Since the optical and $\mathrm{TeV}$ fluxes are well correlated we assumed that both the electron and proton compactnesses should vary according to the optical variability pattern but, in general, with different amplitudes (see eq. (4)). Figure 2 shows our model light curves in the X-ray (first panel), optical (second panel) and $\mathrm{TeV} \gamma$ ray (third panel) energy bands along with the observational data. Using small amplitude variations for all parameters (see Table 1) the variability in the above energy ranges is fairly well reproduced. Moreover, the model predicts no correlation between the optical and $\mathrm{TeV} \gamma$-ray fluxes (fourth panel in Fig. 2). We note that a direct comparison between our model fluxes and the H.E.S.S. data, which are given by [1] in terms of photon count rate per effective area of the detector, could not be made. Thus, we compared their relative variations, i.e. $F_{\mathrm{TeV}} / F_{\mathrm{TeV}}^{\max }$ and $C R / C R^{\max }$, where $C R$ is the photon count rate per effective area of the detector; this is shown in the third panel of Fig. 2. Since this comparison is meaningful only in the absence of spectral changes it cannot be applied in the Fermi data. We

1. We performed a Pearson's correlation test between the optical (BV) and X-ray fluxes. We found a Pearson's correlation coefficient $r(10)=-0.166, p=0.6$, which implies that there is not a statistically significant linear relationship between optical and X-ray fluxes; see also Fig. 1 in [1]. 

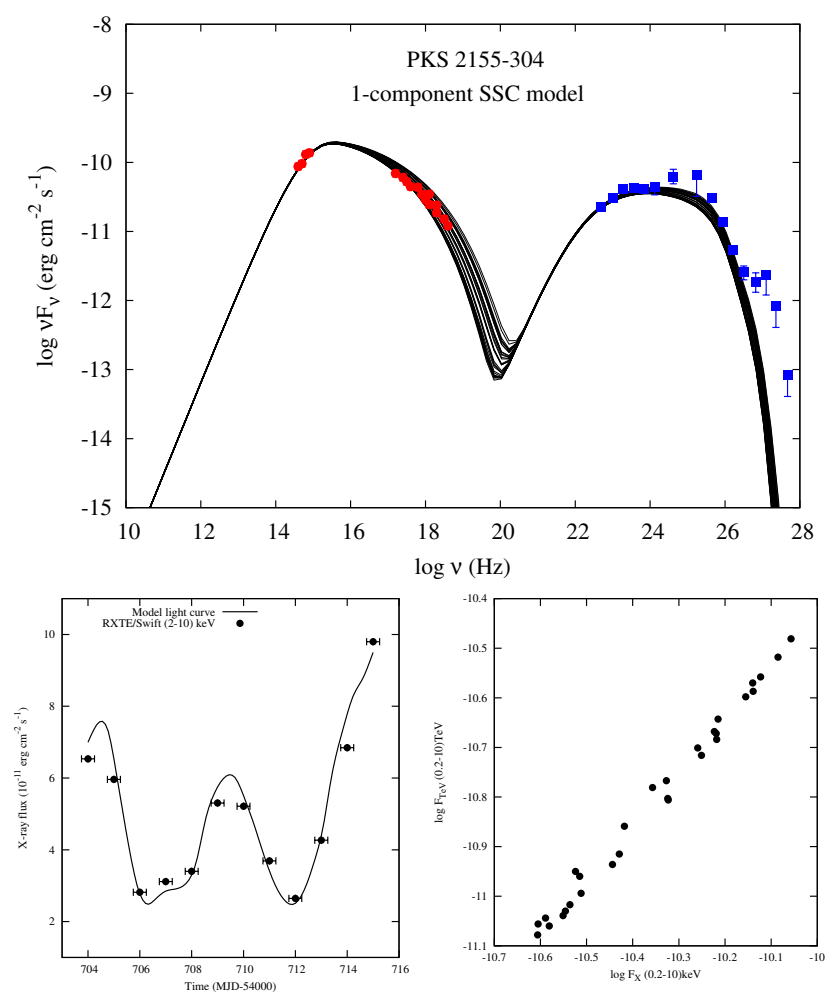

Figure 1. Left panel: Multiwavelength time dependent spectra during the period 54704-54715 MJD. Simultaneous observations with ATOM, RXTE, Fermi and H.E.S.S. (low-to-high frequencies) are shown with points. Bottom left panel: X-ray model lightcurve (solid line) and RXTE/Swift observations (points). Bottom right panel: Log-log plot of the TeV-flux versus the X-ray flux obtained by our model.
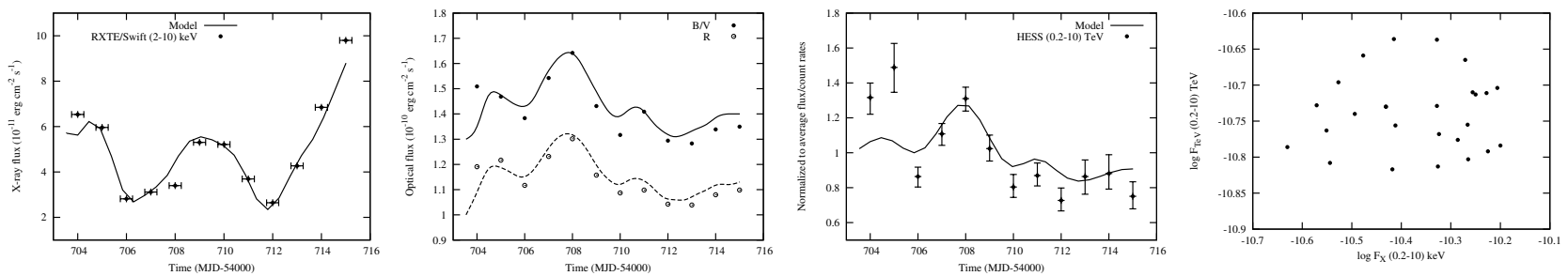

Figure 2. From left to right: X-ray model lightcurve (solid line) and RXTE/Swift observations (points); Optical model lightcurves (BV: solid line; R: dashed line) and ATOM data (BV: filled circles; R: open circles); Plot of the normalized model TeV flux (solid line) and H.E.S.S. photon count rate (points) with respect to their time-averaged values; Log-log plot of the TeV-flux versus the X-ray flux obtained by our model.

note, however, that the (0.2-300) GeV flux given by our model is approximately constant - the relative change of the GeV (logarithim) flux with respect to its time-averaged value is $\sim 0.01$.

Despite the fairly good description of the data by our results, the LHs model requires fine tuning of three model parameters $\left(\gamma_{\max }^{e}, \ell_{e}\right.$ and $\left.\ell_{p}\right)$ which is translated into determining six free parameters (see also Table 1).

\subsection{Two-component SSC model}

Motivated by the uncorrelated variability observed in the optical and X-ray bands, which is difficult to be explained within a single-component emission model (some of the problems were already discussed in $\S 3.1$ ), we con- sidered a two-component SSC scenario. Synchrotron radiation from the first and second components accounts for the X-ray and optical emission, respectively. The highenergy bump of the SED is attributed to the SSC emission from the second component. On the other hand, the parameters describing the first component were chosen in order to supress its SSC emission, as it can be seen by the first panel of Fig. 3. Here, the observed flux variability is modelled by varying the maximum Lorentz factor of electrons according to eqs. (1) and (2) for the first and second component, respectively. In general, the conclusions drawn from our fitting procedure are the same as for the LHs model, i.e. the time-averaged SED and flux variability in optical, $\mathrm{X}$-rays and $\mathrm{TeV} \gamma$-rays are well reproduced at the cost of a large number of parameters. 

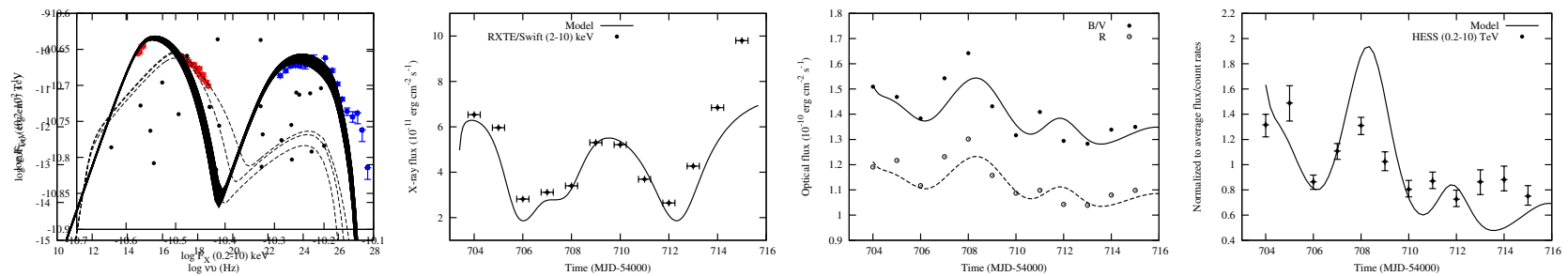

Figure 3. From left to right: Multiwavelength time dependent spectra during the period 54704-54715 MJD. Simultaneous observations with ATOM, RXTE, Fermi and H.E.S.S. (low-to-high frequencies) are shown with points, in addition to the snapshots of the MW emitted by the first (dashed lines) and second (solid lines) components; X-ray model lightcurve (solid line) and RXTE/Swift observations (points); Optical model lightcurves (BV: solid line; R: dashed line) and ATOM data (BV: filled circles; R: open circles); Plot of the normalized model TeV flux (solid line) and H.E.S.S. photon count rate (points) with respect to their time-averaged values.

\section{Discussion}

We have attempted a MW time-dependent fit to blazar PKS 2155-304 while in a low state. Clearly an onecomponent emission model (here, we considered the onezone SSC model), cannot account for the small amplitude variability observed in the optical, $\mathrm{X}$-ray and $\mathrm{TeV} \gamma$-ray energy bands. We have shown that two-component emission models, i.e. LHs and 2-SSC models, are more adequite in reproducing the observed SED and light curves. In particular, the two-component SSC model is prefered over the leptohadronic one due to the smaller number of free parameters required for fitting the observed variability.

In general, our results suggest that, at a low state, the SED of the source might be composed by emission of two or even more components. Thus, a loose correlation between the various energy bands is expected. However, at a high state, e.g. during a flare, only one component lights up and this produces the tight correlations observed in flaring blazars. Concluding, we believe that the two-component emission scenario should be further studied during highstates of blazar emission and we leave this as the subject of a future work.

\section{Acknowledgements}

This research has been co-financed by the European Union (European Social Fund-ESF) and Greek national funds through the Operational Program "Education and Lifelong Learning" of the National Strategic Reference Framework (NSRF) - Research Funding Program: Heracleitus II. Investing in knowledge society through the European Social Fund.

\section{References}

[1] Aharonian F. et al., ApJL, 696, L150-L155 (2009)

[2] Krawczynski H. et al., MNRAS, 336, 721-735 (2002)

[3] Cerutti M. et al., AIPC, 1505, 635-638 (2012)

[4] Dimitrakoudis S. et al., A\&A, 546, 120D (2012)

[5] Mastichiadis A. et al., MNRAS, 434, 2684M (2013) 